\title{
High dose-rate Intra-Operative Radiation Therapy During High Risk Genitourinary Surgery: Initial Observations and a Proposal for its Study in Bladder Cancer
}

\author{
Max Kates*, Meera R. Chappidi, Aaron Brant, Niv Milbar, Nikolai A. Sopko, Christian Meyer, \\ Stephanie A. Terezakis, Joseph M. Herman, Jonathan E. Efron, Bashar Safar, Phuoc T. Tran, \\ Nita Ahuja, Phillip M. Pierorazio and Trinity J. Bivalacqua \\ The James Buchanan Brady Urological Institute and Department of Urology, The Johns Hopkins School of \\ Medicine, Baltimore, MD, USA
}

\begin{abstract}
.
Background: High dose-rate Intra-Operative Radiation Therapy (HD-IORT) is used to provide effective local control for patients with high-risk locally advanced or recurrent tumors. However, the utility of HD-IORT for patients with bladder cancer has not been studied.

Objective: To characterize our institutional experience with HD-IORT in patients with cancer requiring genitourinary surgery, in an effort to identify patients with bladder cancer that may benefit from HD-IORT.

Methods: We performed a retrospective review of all patients who have undergone HD-IORT during genitourinary surgery at our institution. Patients were stratified by surgical margin status, and primary outcomes assessed were overall survival, recurrence free survival and 90-day complications. Patients undergoing cystectomy and HD-IORT with sarcomatoid urothelial cancer were compared to a similar cohort undergoing cystectomy alone. A sample case of one such patient is discussed in detail.

Results: 84 patients at our institution have undergone HD-IORT with genitourinary surgery. Positive surgical margin status was the greatest predictor of both OS $(H R=3.42)$ and RFS $(H R=2.61)$. The overall 90-day complication rate was $61 \%$, with wound infections (43\%) and GI complications (21\%) being most common. 4 of these patients had sarcomatoid urothelial histology, and all are still alive with $>2$ yrs follow up. This compares to a $52 \% 1 \mathrm{yr}$ survival in our sarcomatoid urothelial cohort (25 pts) that did not undergo HD-IORT.

Conclusions: Our institutional experience with HD-IORT has been promising, particularly among patients with locally advanced disease and sarcomatoid histology. We are currently enrolling patients in a multi-institutional registry to assess the utility of HD-IORT in high risk bladder cancer.
\end{abstract}

Keywords: Bladder cancer, radiation therapy

\section{BACKGROUND}

*Correspondence to: Max Kates, The James Buchanan Brady Urological Institute and Department of Urology, The Johns Hopkins School of Medicine, 1800 Orleans Street, Marburg 420, Baltimore, MD 21287, USA. E-mail: mkates@jhmi.edu.
Bladder cancer is a worldwide problem, with nearly 2.7 million people currently suffering with the disease worldwide [1]. Nearly $30 \%$ of these patients present with muscle invasive disease, requiring 
radical cystectomy with pelvic lymph node dissection and urinary diversion $[1,2]$ Despite radical extirpative surgery, $5 \mathrm{yr}$ recurrence free survival is just $68 \%$ among all patients. The most important prognostic indicator is the disease stage, specifically whether the cancer is organ confined and node negative ( 5 yr RFS $85 \%$ ) or extravesical and node negative (RFS 58\%) or node positive (RFS 30\%) [3]. 90\% of recurrences occur within the first two years, and when recurrence is found, median post-recurrence survival is less than 6 months [4]. Pelvic recurrence occurs in up to $20 \%$ of high risk patients, and these patients are often treated with chemotherapy and pelvic radiation [5].

High dose rate intraoperative radiation therapy (HD-IORT) has been utilized to obtain local control for tumors that are unresectable, recurrent, or high risk for local recurrence following primary surgical treatment. HD-IORT can improve local control in multiple cancers, particularly sarcomas and locally advanced rectal and gynecologic cancers $[6,7]$ IORT is discussed as an option in the NCCN guidelines for locally advanced or recurrent rectal, uterine, and cervical cancer [8-10]. Prior efforts to adopt IORT in the treatment of bladder cancer have included intraoperative radium brachytherapy and external beam radiation [11-14]. However many of treatments were attempted in an earlier era of radiation therapy, and currently IORT is not standard practice at the time of cystectomy for bladder cancer. Radiation therapy is increasingly being utilized with chemotherapy and aggressive TURBT in the treatment of muscle invasive therapy for select patients, and there is a rationale for combined use with surgery in patients at high risk for local recurrence. Since 2006, our institution has performed HD-IORT utilizing brachytherapy for high-risk pelvic and retroperitoneal soft tissue malignancies. Many of these cases have included the removal of genitourinary organs and the inclusion of urologic oncologists within the interdisciplinary team [15]. However, the utility of HD-IORT in patients with genitourinary involvement is unknown. High risk bladder cancer shares much in common with other locally aggressive pelvic malignancies, and HD-IORT may have a logical role for certain cases. Our objective was to determine the safety and efficacy of intra-operative radiation therapy at the time of genito-urinary surgery either alone or when performed concurrently with other high risk oncologic surgeries. We then seek to outline parameters for the study of HD-IORT at the time of radical cystectomy for patients with urothelial carcinoma with high risk features.

\section{METHODS}

\section{Patient selection}

Patients at our institution from 2006-2016 who received HD-IORT during surgery for tumors with GU involvement were identified. Patients who were thought to benefit from HDR-IORT have been previously discussed, but include 1) pre-operative imaging concerning for close or positive margins and 2) The dose of adjunctive EBRT cannot be obtained due to proximity to surrounding structures including bowel and bladder. Selected patients meet with several members of the HD-IORT team, including urologic and surgical oncology, radiation oncology, and anesthesia; several more team members are also involved including a nurse coordinator, medical physicist, and surgical pathologist (Fig. 1). A checklist for

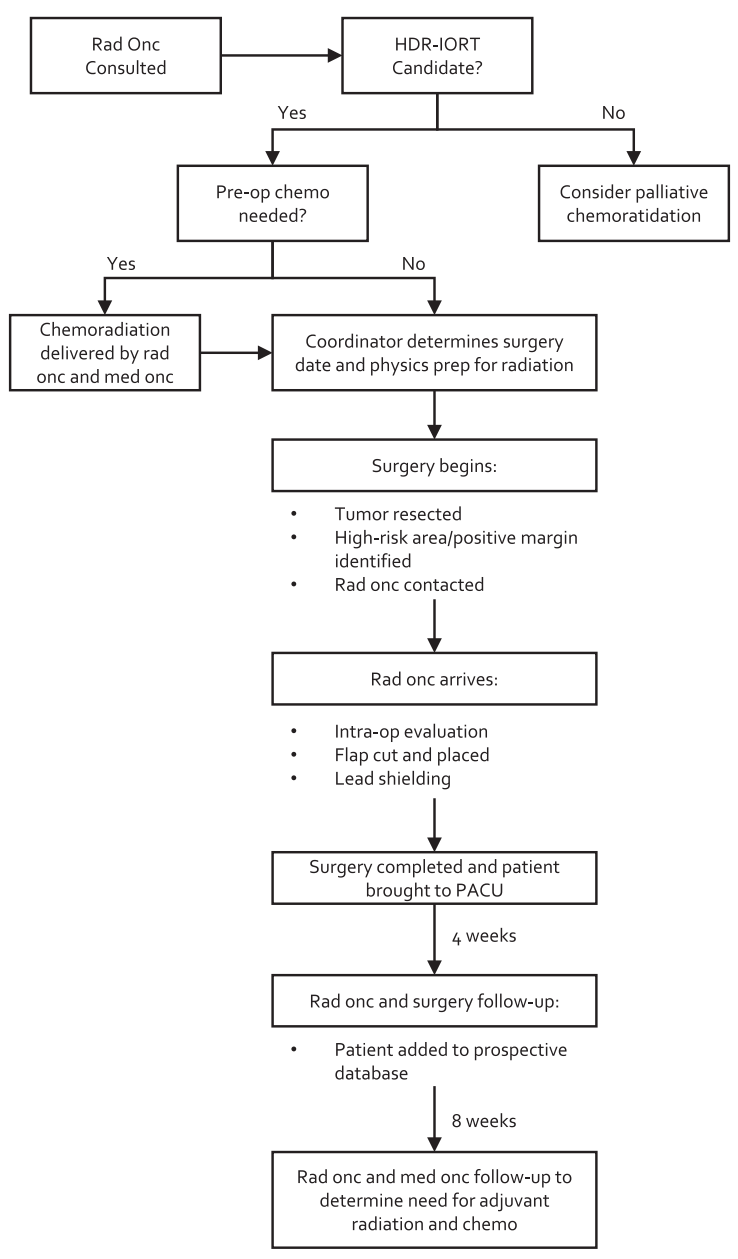

Fig. 1. The HD-IORT flowchart which depicts the logistical undertaking of the JHH HD-IORT program. 


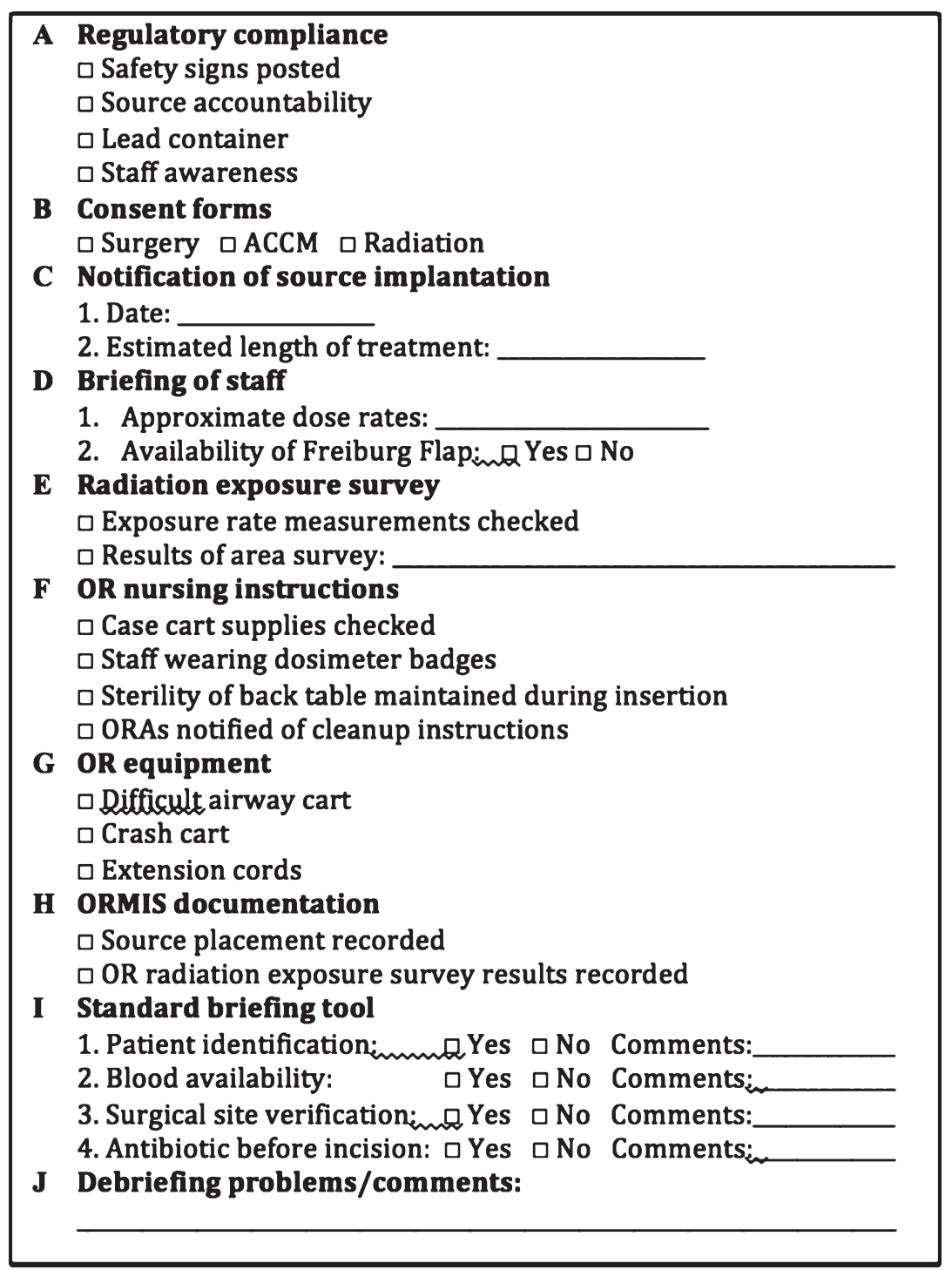

Fig. 2. HD-IORT institutional checklist.

HD-IORT has been in place at our institution for several years to minimize risk and ensure proper training and collaboration between teams [16] (Fig. 2).

\section{Intervention}

IORT: We have previously described our HDRIORT program, but briefly, following tumor resection and prior to urinary and GI tract reconstruction, the high-risk area of the surgical bed is measured and flexible flap applicator (Freiburg Flap ${ }^{\circledR}$, Nucletron, an Elekta company, Elekta AB, Stockholm, Sweden) consisting of linear, connected 1-cm silicone rubber balls forming a planar flexible sheet is cut to the size of the measured target area with possibly additional margin to cover suspected subclinical/high-risk regions (Fig. 3) [15]. Prior to placement of the flap 6-French afterloading catheters are threaded through and numbered to correspond to a specific channel of the HDR afterloader unit. The flap apparatus with attached catheters is then placed against the area of interest with lap sponges. Lead shields are used overlying the bowel and ureters for protection and covered with wet gauze to prevent backscatter. As has been described, lead shielding can reduce the dose delivered to normal tissue by $70 \%$. This customized flap delivers a uniform dose of radiation via an encapsulated 192Ir source, half-life of 73 days, 
which is loaded via 6-French afterloading catheters. The dose is routinely $10-12 \mathrm{~Gy} \times 1$, and $>12$ Gy in the case of macroscopic residual disease. Other predominant methods of IORT for treatment of pelvic tumors reported in the literature are electron-based or with orthovoltage units. The relative merits and disadvantages of each have been discussed previously [17-19].

\section{Clinical cohort}

Of 125 patients who underwent IORT since 2006, $84(67 \%)$ required urological surgery. Three of the patients had two resections requiring urology surgery. For survival analysis the date of the first surgery was the start of follow-up. For complication data following surgery both resections were used $(n=87)$. Of the 84 cases, $46(55 \%)$ were for treatment of sarcoma (10 of primary GU origin), 13 (21\%) for colorectal carcinoma, and $8(13 \%)$ for varying gynecologic or anal malignancies. Of the 87 surgeries performed, patients underwent radical cystectomy with urinary diversion in 33 (38\%) of cases (Fig. 4). Of the 16 cases of primary genito-urinary origin, 6 were bladder tumors, of which 4 were carcinosarcoma/sarcomatoid urothelial carcinoma.

\section{Statistics}

Patients undergoing IORT were stratified by surgical margin status. Baseline sociodemographic variables were compared with chi-squared and student $t$-test where appropriate. For the purposes of assessing IORT, recurrence was defined as local recurrence in the pelvis. We were particularly interested in the experience of IORT in the setting of positive and negative surgical margins, and thus performed Kaplan-Meir curves to compare OS and RFS between these two groups (Fig. 5). Univariate and multivariate cox proportional hazards models were utilized to identify predictors of OS and RFS. Variables examined included age, positive margin status upon resection, and receipt of neoadjuvant chemotherapy. For the OS model, local recurrence was included. In an effort to specifically look at our experience treating sarcomatoid urothelial carcinoma with HD-IORT, we compared the patients with primary sarcomatoid variant of the bladder who underwent HD-IORT with radical cystectomy with patients undergoing cystectomy for sarcamtoid urothelial carcinoma without IORT. All reported $p$-values two-sided with $p$-value $\leq 0.05$ as statistically significant.

\section{RESULTS}

Mean IORT dose administered was $11.8 \mathrm{~Gy}$ (range 10-20 Gy). Both neoadjuvant (36\%) and adjuvant $(15 \%)$ chemotherapy regimens were utilized. With a median follow up of 21 months (mean follow up 26 months), 2 yr survival is $65 \%$. Positive margins were associated with worse OS $(p=0.049)$ and RFS $(p=0.02)$ (Fig. 5A, B). In a multivariate cox-porportional hazards model, positive margins were associated with worse OS (HR 3.59; 95\%CI 1.36-9.46) and RFS (HR 2.61; 95\% CI $1.17-5.84)$.

Of $56(64 \%)$ patients who experienced a Clavien 1-5 complication, wound infection (43\%), gastrointestinal complication including ileus $(21 \%)$, and neuropathy $(13 \%)$ were most common. Thus far, there have been no fistula formations, breakdowns of anastomoses (either bowel or ureteral) or delayed ureteral injuries with the use of IORT. Complications were fairly consistent between patients with primary GU pathology and those with other pathology (Fig. 6). Of the patients who underwent HD-IORT with cystectomy for any cause, complications were observed in $23(70 \%)$, with $7(21 \%)$ being Clavien $\geq 3$ postoperative.

29 patients at JHH over the study period had sarcomatoid urothelial carcinoma, of whom 4 underwent HD-IORT with cystectomy and 25 underwent cystectomy alone. Mean age at surgery was 69 years, and $24 \%$ underwent neoadjuvant chemotherapy. Patients were more likely to have pathologic T3 (44\%vs 26\%) or T4 (31vs 10\%) disease, and N1-2 disease (24\%vs $18 \%$ ) compared to conventional urothelial carcinoma $(p<0.001)$. Among patients not receiving IORT, one year local recurrence occurred in 56\% of patients at a median time to recurrence of 180 days. Following recurrence, median time to death was 60 days. One year overall survival was $52 \%$. Of the 4 cases treated with IORT thus far, there have been no local recurrences, and 1 case of a distant metastasis, with all patients currently alive with at least 2 years of follow up.

\section{Case study: Locally advanced sarcomaoid urothelial carcinoma}

A 72-year old female with a 50 pack-year smoking history presented to her primary care doctor with increasing urinary frequency, urge incontinence, and gross hematuria, which prompted a urologic workup. Cystoscopy demonstrated a large left sided bladder 


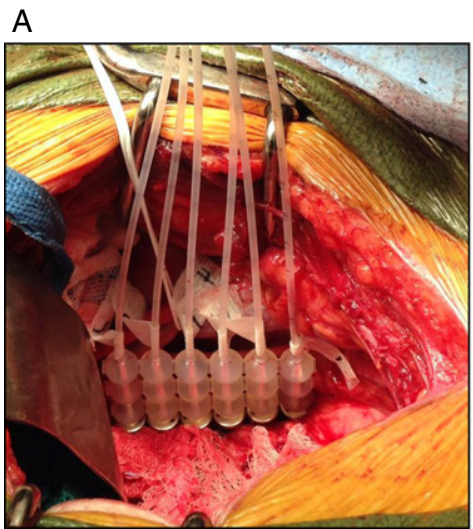

B

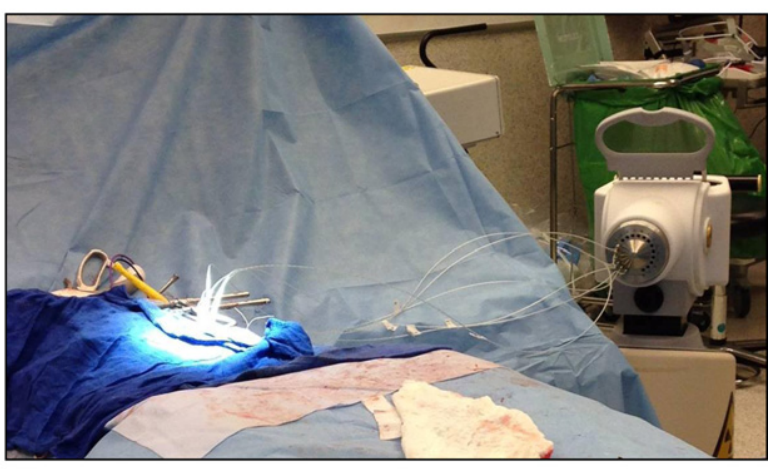

Fig. 3. Following resection, (A) the area of interest is isolated from surrounding bowel and ureter, and (B,C) a Freiberg flap is placed with flexible beads and $6 \mathrm{Fr}$ catheters connecting to the afterloader.

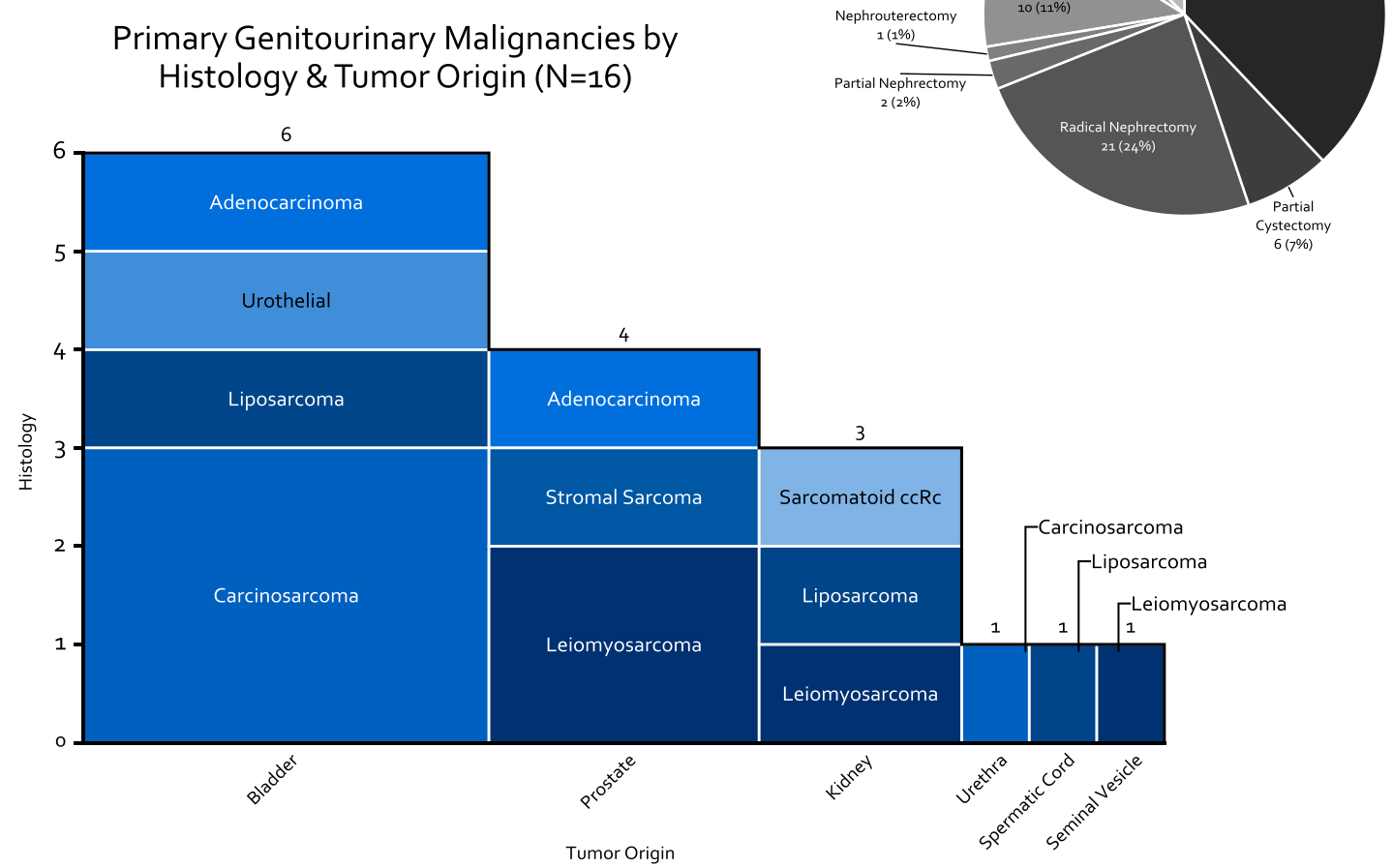

Fig. 4. Breakdown of HD-IORT Cases by type of surgery, disease location, and histology.

tumor, and CT imaging demonstrated moderate left hydronephrosis, and a left posterior bladder mass with loss of the fat plane between the bladder and vagina (Fig. 7). The patient was referred to our clinic, and underwent transurethral resection of a $5 \mathrm{~cm}$

Total IORT Cases with Genitourinary Surgery $(\mathrm{N}=87)$

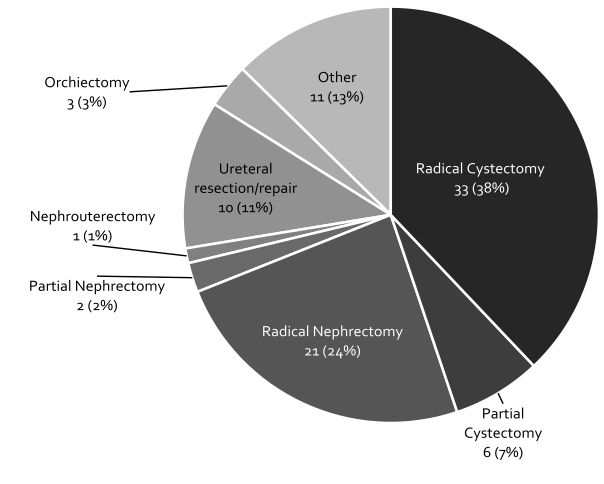


A

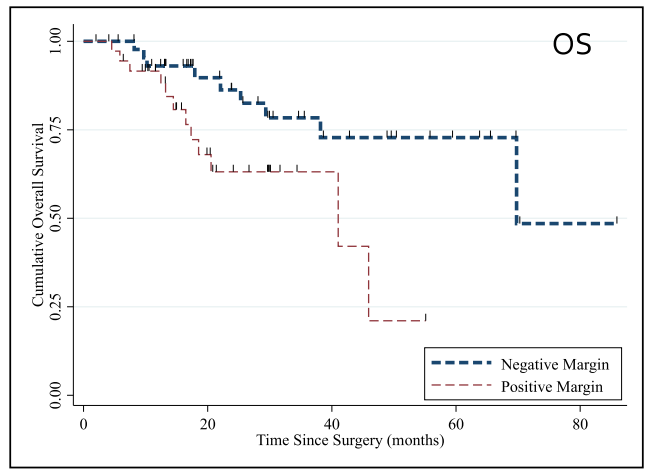

Number At Risk Negative Margi Positive Margin
B

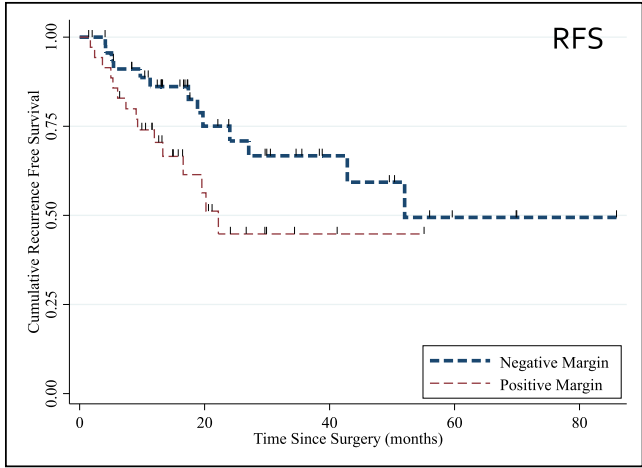

Number At Risk Negative Margin
Positive Margin

Fig. 5. (A-B) Kaplan Meier Curve for OS $(p=0.049)$ and RFS $(p=0.02)$ stratified by surgical margin status.

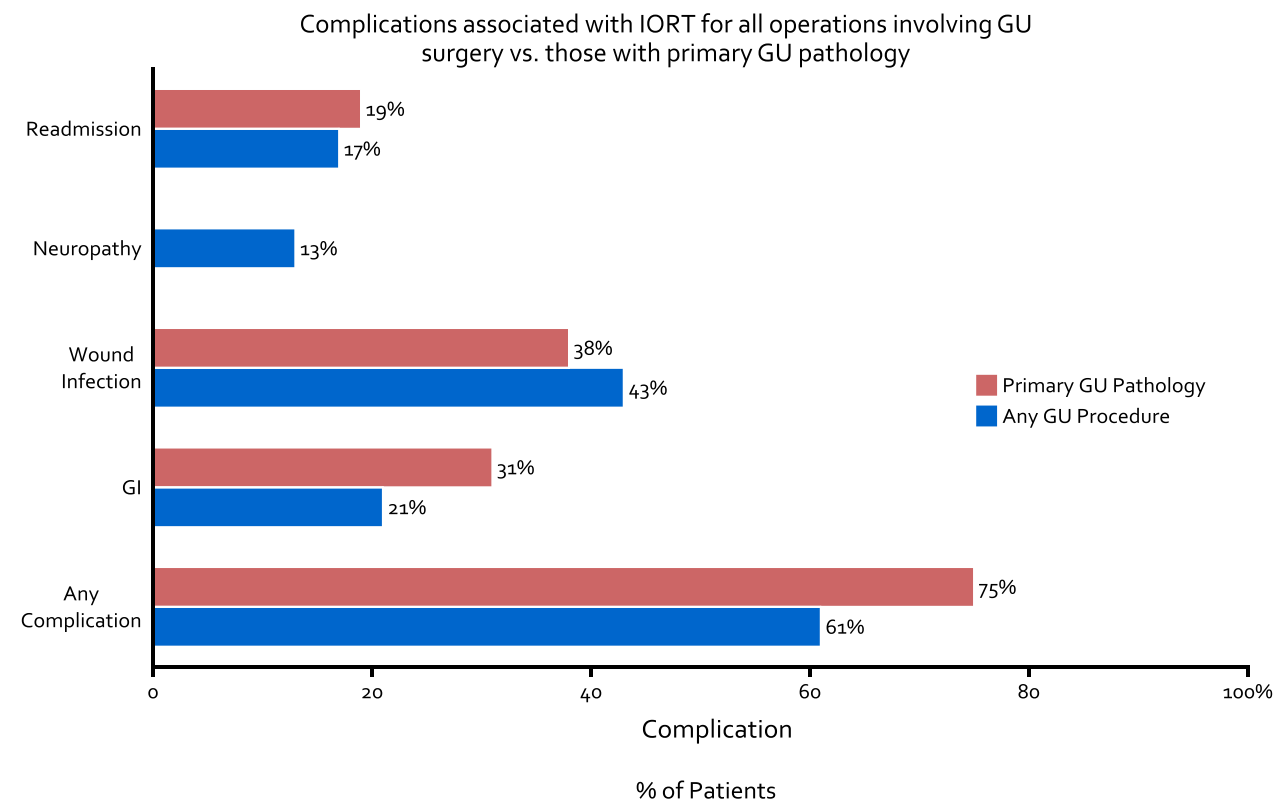

Fig. 6. Complications associated with IORT for all operations involving GU surgery (blue bar) as well as those with primary GU pathology (red bar).

Approximately 1 month after initial presentation, the patient underwent anterior exenteration, a bilateral pelvic lymph node dissection, HD-IORT, and ileal conduit urinary diversion. There was concern for tumor extending beyond the bladder laterally and intraoperative frozen sections were obtained of the left pelvic sidewall which was negative for cancer. A $4 \times 8 \mathrm{~cm}$ Freiberg flap was then placed in the tumor bed, and a total dose of $12 \mathrm{~Gy}$ was delivered to the left pelvic sidewall and obturator fossa. All margins were negative and final pathology demonstrated pT3b, N0 sarcomatoid urothelial carcinoma invading the periversicular tissue. The patient had an uneventful hospital course and was discharged from the hospital on postoperative day 7 . The patient has been doing well since surgery with no evidence of recurrences or complications at a follow-up time of 42 months.

\section{DISCUSSION}

Our institutional experience with HD-IORT has been promising, particularly among patients with locally advanced disease and sarcomatoid histology. 

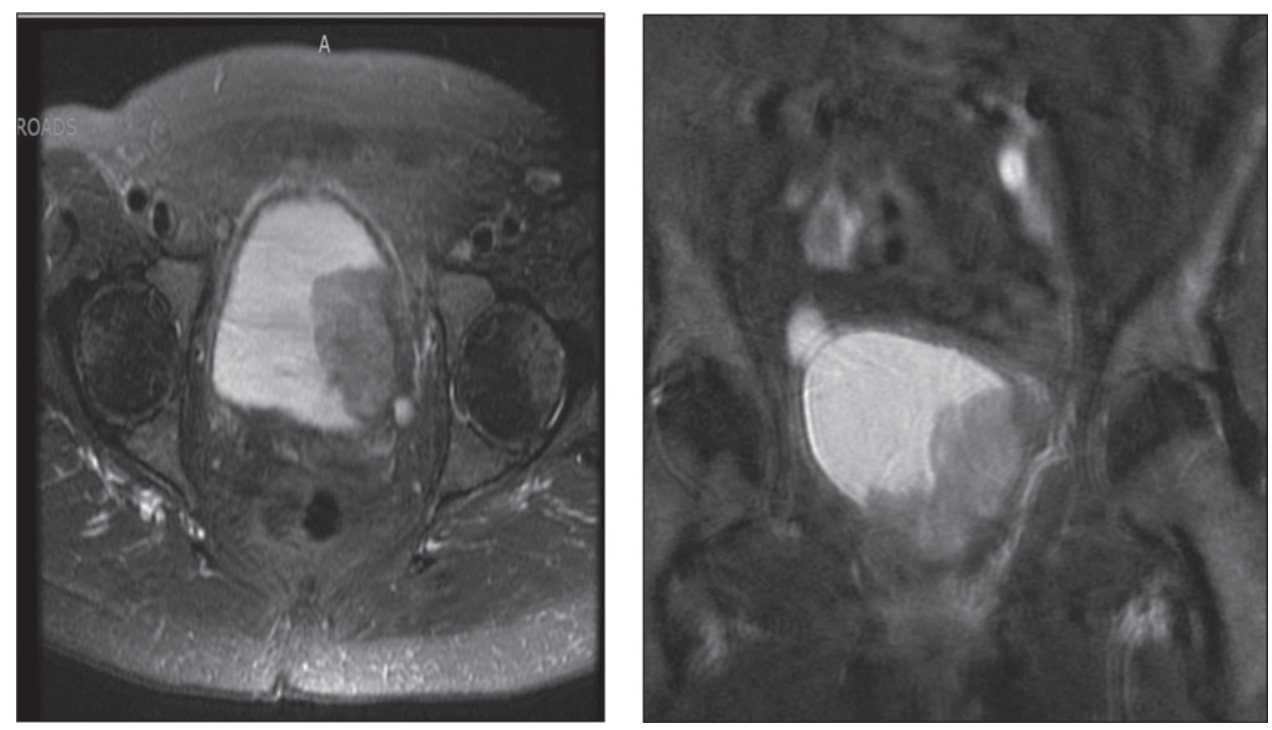

Fig. 7. T2 weighted contrast enhanced MRI in axial and coronal views demonstrating bladder tumor appearing to extend into the left perivesical fat.

Ultimately the goal of radiation at the time of resection is to establish local control of tumors at high risk for recurrence. All prior studies attempting to improve local pelvic control using an IORT "boost" have included the use of external beam radiation therapy; however this study utilizes high dose brachytherapy. Local recurrence after cystectomy is currently associated with a median survival of 8 months when presenting without distant mets, and 4 months when presenting concurrently with distant mets [4]. The sarcomatoid urothelial variant is relatively rare, and is particularly associated with abysmal survival, with a $1 \mathrm{yr}$ survival less than $50 \%$ in multiple series [20-22]. We believe that this variant warrants aggressive local control, and currently offer the majority of these patients neoadjuvant chemotherapy with cisplatin, gemcitabine, and docetaxol with HD-IORT when feasible. Our chemotherapy regimen for sarcomatoid urothelial carcinoma is based on data supporting gemcitabine and docetaxol for sarcoma, and its use for sarcomatoid urothelial bladder cancer is being actively investigated [23]. We also believe that HD-IORT may benefit tumors with aggressive clinical and molecular features that are at a high risk of recurring locally. It should be noted however that patients with gross positive margins appear to have very poor survival even with HD-IORT, and thus obtaining negative gross margins trumps all other adjunctive therapies.

It should be noted that patients treated with HDIORT may have slightly more complications than patients undergoing cystectomy at our institution and elsewhere [24-26]. Wound infections and pelvic abscesses in particular appear to be greater when HDIORT is used, although characterizing this increased risk needs to be done in future studies. It should be noted that the colorectal surgeons at our institution initiated a comprehensive program to reduce surgical site infections during the study period, which was subsequently adopted by the urologic oncologists [27]. One of the main principles of this effort is utilizing negative pressure "incisional vac" devices, which were first described for hernia repair at our institutions and are now routinely used in complex cases at high risk for wound infection [28]. Chemotherapy may play a role in this as well. Additionally, a limitation of the current study is that chemotherapy regimens, dose reduction, and timing were unavailable as many patients receive this chemotherapy outside of the institution. Another study limitation is the lack of a well matched historical cohort with positive margins to serve as a comparator. As we accrue more patients, we plan to directly compare IORT with observation in the setting of positive surgical margins, as our data suggests that patients with positive margins do poorly regardless of the adjuvant therapy employed. Understanding which patients may benefit from HD-IORT is thus paramount, in order to maximize local control of aggressive variants while minimizing added toxicity.

HD-IORT should not be considered standard of care, nor should it be encouraged outside of tertiary 
care centers experience in its delivery. In an effort to better understand a potential role for HD-IORT in our high risk bladder cancer patients, we have initiated a multi-institutional registry to enroll and follow these patients in a prospective fashion. Our inclusion criteria include adult patients aged $>18$ with 1) T3/T4 on preoperative imaging/ Bimanual concerning for fixation to pelvic sidewall 2) T2 with sarcomatoid urothelial carcinoma (carcinosarcoma) or 3)Patients with squamous cell carcinoma (SCC). Immediately routine post-operative care will be initiated. As is routine, if patients have aggressive pathologic features (Pt3-4, N+) they will meet with a medical oncologist even if they received neoadjuvant chemotherapy prior to cystectomy. Patients will have routine axial imaging with CT every 3 months for 2 years, followed by every 6 months for 5 yrs and yearly therafter. Outcomes of interest include toxicity and post-operative complications, pelvic recurrence and general recurrence free survival, and overall survival.

Finally, it should be noted that the field of bladder cancer is quickly evolving in the age of immunotherapy, and this evolution brings with it new possibilities for treating aggressive and locally advanced disease. The most important advance for treating cancer in the last decade has been the discovery, development, and approval of immune checkpoint inhibitors, which have become central components of multimodal cancer therapy [29]. Bladder cancer is one of the most immunogenic cancers, and atezolizumab, a PD-L1 checkpoint inhibitor, has already gained FDA approval for advanced bladder cancer-the first drug approval for this disease in 3 decades. We are at the forefront of an exciting time in immunotherapy drug development.

The Abscopal Effect suggests that radiation delivered locally can minimize or eradicate distant metastases [30]. The immune system has been shown to be a crucial mediator of the Abscopal Effect, which has led to a renewed interest in combination immunotherapy with radiation [31]. Indeed, there are several clinical trials combining immune checkpoint inhibitors with radiation therapy for multiple cancers, including a Phase 1 trial for invasive bladder cancer (NCT02560636) [32]. One can foresee a future where a patient with clinical T3/T4 bladder cancer undergoes neoadjuvant chemotherapy, has stable disease or disease progression, undergoes radical cystectomy with IORT and adjuvant immunotherapy. The opportunities are vast, but much work lies ahead to maximize therapy for patients with high risk bladder cancer.

\section{CONCLUSIONS}

HD-IORT is a safe and feasible treatment for high-risk patients undergoing complex surgery for sarcomas and other soft tissue malignancies with GU involvement. While IORT can improve local control, positive surgical margin status is still an important predictor of both OS and RFS. In well-selected patients with sarcomatoid urothelial carcinoma, IORT may help prevent or delay local recurrence. As HD-IORT expands into urologic oncology, future studies are needed to address the benefit of HD-IORT vs. additional local toxicity A multi-institutional effort is currently underway to investigate the role of HD-IORT at the time of cystectomy for high-risk bladder cancer.

\section{CONFLICT OF INTEREST}

The authors have no conflict of interest to report.

\section{DISCLOSURES}

Nothing to disclose.

\section{REFERENCES}

[1] Siegel RL, Miller KD, Jemal A. Cancer statistics, 2015. CA Cancer J Clin [Internet] 2015 [cited 2015 Jan 9];65(1):5-29. Available from: http://www.ncbi.nlm.nih. gov/pubmed/25559415

[2] Ploeg M, Aben KKH, Kiemeney LA. The present and future burden of urinary bladder cancer in the world. World J Urol 2009;27(3):289-93.

[3] Stein JP, Lieskovsky G, Cote R, Groshen S, Feng AC, Boyd S, et al. Radical cystectomy in the treatment of invasive bladder cancer: Long-term results in 1,054 patients. J Clin Oncol [Internet] 2001;19(3):666-75. Available from: http://www.ncbi.nlm.nih.gov/pubmed/11157016

[4] Mitra AP, Quinn DI, Dorff TB, Skinner EC, Schuckman AK, Miranda G, et al. Factors influencing post-recurrence survival in bladder cancer following radical cystectomy. BJU Int 2012;109(6):846-54.

[5] Westney OL, Pisters LL, Pettaway CA, Tu SM, Pollack A, Dinney CP. Presentation, methods of diagnosis and therapy for pelvic recurrence following radical cystectomy for transitional cell carcinoma of the bladder. J Urol 1998;159(3):792-5.

[6] Harrison LB, Minsky BD, Enker WE, Mychalczak B, Guillem J, Paty PB, et al. High dose rate intraoperative radiation therapy (HDR-IORT) as part of the management strategy for locally advanced primary and recurrent rectal cancer. Int J Radiat Oncol Biol Phys 1998;42(2):325-30.

[7] Niewald M, Fleckenstein J, Licht N, Bleuzen C, Ruebe C. Intraoperative radiotherapy (IORT) combined with external beam radiotherapy (EBRT) for soft-tissue sarcomas - A retrospective evaluation of the Homburg experience in the years 1995-2007. Radiat Oncol 2009;4:32. 
[8] NCCN. NCCN Clinical Practice Guidelines in Oncology: Uterine Neoplasms. Nccn [Internet]. 2015; Available from: http://www.nccn.org/professionals/physician_gls/pdf/ uterine.pdf

[9] NCCN. NCCN Clinical Practice Guidelines in Oncology (NCCN Guidelines $\left.{ }^{\circledR}\right)$ Rectal Cancer [Internet]. Version 2.2016. 2016. pp. 1-136. Available from: https://www.nccn. org/professionals/physician_gls/pdf/rectal.pdf

[10] National Comprehensive Cancer Network. NCCN Clinical Practice Guidelines in Oncology: Cervical Cancer.V 1.2016. J Natl Compr Canc Netw [Internet] 2016;64. Available from: https://books.google.com/books?id=ZeQvPeSG SOgC\&pgis $=1$

[11] Van der Werf-Messing BHP. Cancer of the urinary bladder treated by interstitial radium implant. Int $\mathbf{J}$ Radiat Oncol [Internet] 1978 [cited 2017 Jun 19];4(5-6):3738. Available from: http://linkinghub.elsevier.com/retrieve/ pii/0360301678900652

[12] Nieuwenhuijzen JA, Horenblas S, Meinhardt W, van Tinteren H, Moonen LMF. Salvage cystectomy after failure of interstitial radiotherapy and external beam radiotherapy for bladder cancer. BJU Int [Internet]. Blackwell Science Ltd 2004 [cited 2017 Jun 19];94(6):793-7. Available from: http://doi.wiley.com/10.1111/j.1464-410X.2004.05034.x

[13] Abe M, Takahashi M. Intraoperative radiotherapy: The japanese experience. Int J Radiat Oncol [Internet] 1981 [cited 2017 Jun 19];7(7):863-8. Available from: http:// linkinghub.elsevier.com/retrieve/pii/0360301681900018

[14] Intraoperative and External Beam Radiotherapy in Invasive B1...: American Journal of Clinical Oncology [Internet] [cited 2017 Jun 19]. Available from: http://journals.lww. com/amjclinicaloncology/abstract/1990/04000/intraopera tive_and_external_beam_radiotherapy_in.3.aspx

[15] Moningi S, Armour EP, Terezakis SA, Efron JE, Gearhart SL, Bivalacqua TJ, et al. High-dose-rate intraoperative radiation therapy: The nuts and bolts of starting a program. Vol. 6, Journal of Contemporary Brachytherapy 2014:99-105.

[16] Rodriguez-Paz JM, Mark LJ, Herzer KR, Michelson JD, Grogan KL, Herman J, et al. A novel process for introducing a new intraoperative program: A multidisciplinary paradigm for mitigating hazards and improving patient safety. Anesth Analg 2009;108(1):202-10.

[17] Mirnezami R, Chang GJ, Das P, Chandrakumaran K, Tekkis $\mathrm{P}$, Darzi A, et al. Intraoperative radiotherapy in colorectal cancer: Systematic review and meta-analysis of techniques, long-term outcomes, and complications. Surg Oncol [Internet] 2012. Available from: http://www.ncbi.nlm.nih. gov/pubmed/23270946

[18] Piontek RW, Kase KR. Design and dosimetric properties of an intraoperative radiation therapy system using an orthovoltage $\mathrm{x}$ ray unit. Int $\mathrm{J}$ Radiat Oncol Biol Phys 1986;12(2):255-9.

[19] Tran PT, Su Z, Hara W, Husain A, Teng N, Kapp DS. Long-Term Survivors Using Intraoperative Radiotherapy for Recurrent Gynecologic Malignancies. Int J Radiat Oncol Biol Phys 2007;69(2):504-11.

[20] Wright JL, Black PC, Brown GA, Porter MP, Kamat AM, Dinney CP, et al. Differences in survival among patients with sarcomatoid carcinoma, carcinosarcoma and urothelial carcinoma of the bladder. J Urol 2007;178(6):2302-7.

[21] Sanfrancesco J, McKenney JK, Leivo MZ, Gupta S, Elson P, Hansel DE. Sarcomatoid urothelial carcinoma of the blad- der: Analysis of 28 cases with emphasis on clinicopathologic features and markers of epithelial-to-mesenchymal transition. Arch Pathol Lab Med 2016;140(6):543-51.

[22] Young RH, Wick MR, Mills SE. Sarcomatoid carcinoma of the urinary bladder: A clinicopathologic analysis of 12 cases and review of the literature. Am J Clin Pathol [Internet] Oxford University Press 1988 [cited 2017 Jun 19];90(6):653-61. Available from: https://academic.oup. com/ajcp/article-lookup/doi/10.1093/ajcp/90.6.653

[23] Hensley ML. Gemcitabine and docetaxel in patients with unresectable leiomyosarcoma: Results of a phase II trial. J Clin Oncol [Internet] 2002;20(12):2824-31. Available from: http://jco.ascopubs.org/cgi/content/abstract/20/12/2824

[24] Novara G, De Marco V, Aragona M, Boscolo-Berto R, Cavalleri S, Artibani W, et al. Complications and mortality after radical cystectomy for bladder transitional cell cancer. J Urol 2009;182(3):914-21.

[25] Shabsigh A, Korets R, Vora KC, Brooks CM, Cronin AM, Savage C, et al. Defining early morbidity of radical cystectomy for patients with bladder cancer using a standardized reporting methodology. Eur Urol 2009;55(1):164-74.

[26] Chappidi MR, Chalfin HJ, Johnson DJ, Kates M, Sopko NA, Johnson $\mathrm{MH}$, et al. Longer average blood storage duration is associated with increased risk of infection and overall morbidity following radical cystectomy. Urol Oncol [Internet] 2016. Available from: http://www.ncbi.nlm.nih. gov/pubmed/27771280

[27] Wick EC, Hobson DB, Bennett JL, Demski R, Maragakis L, Gearhart SL, et al. Implementation of a surgical comprehensive unit-based safety program to reduce surgical site infections. J Am Coll Surg [Internet] 2012 [cited 2017 Jun 19];215(2):193-200. Available from: http:// linkinghub.elsevier.com/retrieve/pii/S1072751512002645

[28] Soares KC, Baltodano PA, Hicks CW, Cooney CM, Olorundare IO, Cornell P, et al. Novel wound management system reduction of surgical site morbidity after ventral hernia repairs: A critical analysis. Am J Surg [Internet] 2015 [cited 2017 Jun 19];209(2):324-32. Available from: http:// linkinghub.elsevier.com/retrieve/pii/S0002961014003808

[29] Sharma P, Allison JP. The future of immune checkpoint therapy. Science (80-) [Internet] 2015;348(6230):5661. Available from: http://www.sciencemag.org/cgi/doi/ 10.1126/science.aaa8172

[30] Postow MA, Callahan MK, Barker CA, Yamada Y, Yuan J, Kitano S, et al. Immunologic correlates of the abscopal effect in a patient with melanoma. N Engl J Med [Internet] 2012;366(10):925-31. Available from: http://www. pubmedcentral.nih.gov/articlerender.fcgi? artid $=3345206 \&$ tool=pmcentrez\&rendertype $=$ abstract

[31] Tang C, Wang X, Soh H, Seyedin S, Cortez MA, Krishnan $S$, et al. Combining radiation and immunotherapy: A new systemic therapy for solid tumors? Cancer Immunol Res [Internet] 2014;2(9):831-8. Available from: http://www. ncbi.nlm.nih.gov/pubmed/25187273 \nhttp://cancerimmun olres.aacrjournals.org/content/2/9/831.full.pdf

[32] Kang J, Demaria S, Formenti S. Current clinical trials testing the combination of immunotherapy with radiotherapy. J Immunother Cancer [Internet] 2016;4(1):51. Available from: http://jitc.biomedcentral.com/articles/10.1186/ s40425-016-0156-7 